\title{
L'habit ne fait pas le moine: à propos de la fable De lupo qui voluit esse monachus d'Eudes de Cheriton (XIIIème siècle): Quelques réflexions sur la complémentarité de la fable et du proverbe*
}

\section{Do not Judge by Appearances: About the Fable De lupo qui voluit esse monachus of Eudes de Cheriton (tirteenth century): Some Considerations on the Complementarity of the Fable and Proverb}

BERNARD DARBORD

Département d'espagnol

Université de Paris Nanterre

200 Avenue de la République. Nanterre, 92000. Francia

bdarbord@parisnanterre.fr

Orcid ID: 0000-0002-1681-1102

Resumen: El refrán y la fábula remiten a una sabiduría práctica, basada en la modestia, la simplicidad, y la búsqueda de la verdad. Adaptando los modelos de la tradición esópica, los autores medievales han configurado una moralización de las fábulas, defendiendo a los humildes y condenando a los clérigos ambiciosos. La frase sentenciosa y el refrán son, para Odo de Cheriton, una excelente herramienta para la edificación moral.

Palabras clave: Fábula. Refrán. Paradoja. Cinismo. Edad Media. Odo de Cheriton.
RECIBIDO: 20 DE FEBRERO DE 2017 ACEPTADO: 22 DE SEPTIEMBRE DE 2017
Abstract: The proverb and the fable are based on practical wisdom, based on modesty, simplicity and the quest for truth. By adapting the models of the aesopic tradition, medieval authors developed a moralization of fables, defending the humble and condemning ambitious clerics. The proverbial sentence and the proverb are for Eudes de Cheriton an excellent tool of moral edification.

Keywords: Fable. Proverb. Paradox. Cynicism. Middle Ages. Eudes de Cheriton.

\footnotetext{
* Ce travail fait partie du projet FFI2012-32265, "La fábula esópica del siglo XIV", financé par le Ministerio de Economía y Competitividad espagnol.
} 
Résumé: Le proverbe et la fable reposent sur une sagesse pratique, fondée sur la modestie, la simplicité et la quête de la vérité. En adaptant les modèles de la tradition ésopique, les auteurs médiévaux ont développé une moralisation des fables, défendant les humbles et condamnant les clercs ambi- tieux. La phrase sentencieuse et le proverbe sont pour Eudes de Cheriton un excellent outil d'édification morale.

Mots clés: Fable. Proverbe. Paradoxe. Cynisme. Moyen Age. Eudes de Cheriton.

es Disticha Catonis sont un recueil de 144 aphorismes dont les origines sont discutées. En 1950, à tort ou à raison, Léon Herrmann, latiniste belge de renom, les a attribués à Phèdre, le grand fabuliste latin du premier siècle après Jésus-Christ. Cette attribution n'a pas fait l'unanimité de la critique: les distiques de Caton semblent de facture plus récente. Herrmann s'appuyait notamment sur le fait que Pierre Pithou, auteur en 1596 de la première édition des fables de Phèdre, a désigné par Uet. Ex. Cat un manuscrit qui ressemble à celui de Phèdre et contient les distiques en question. L'argument essentiel du philologue belge est que les fables et les maximes partagent une philosophie commune de la vie, fondée sur une morale empreinte de modération et de sagesse pratique, de discrétion aussi. Cette morale pouvait s'exprimer par l'exemple (fables et exempla). Elle pouvait s'exprimer de façon plus lapidaire par la sentence et le proverbe, de fort contenu performatif et injonctif, dénuées de mots inutiles. Ainsi s'achèvent les Distica:

Miraris uersus nudis me scribere uerbis:

Hoc breuitas fecit, sensu uno iungere binos. (II, 49)

'Tu t'étonnes que j'écrive ces vers en mots si nus:

C'est la brièveté qui a été cause que j'accouple deux vers pour une pensée'.

Deux vers qui souvent s'adaptent aux deux membres d'une parémie. Si l'hypothèse d'Herrmann se vérifiait, cela voudrait dire que Phèdre a voulu réunir en une œuvre la fable et le proverbe, comme moyen d'exprimer une même forme de sagesse.

Fable et proverbe, en tout cas, condamnent l'ambition et la présomption, le mensonge et la dissimulation, la convoitise et la jactance. Foris Cato intus Nero (Carron 178) disait un aphorisme du Moyen Age, pour dire l'hypocrisie de certains: 'Néron à l'intérieur, sous des dehors de sage'. N'est-ce pas ce que nous enseigne la fable du "Loup qui se faisait moine" avec laquelle nous illustrons notre étude? Il est en tout cas facile d'observer que le récit de la fable s'aide souvent d'un recours au proverbe pour dire et redire une forme simple 
de sagesse, sans doute héritée des philosophes cyniques, comme l'a défendu Rodríguez Adrados sur qui nous nous appuyons (voir plus bas notre note 4).

Nous nous servirons essentiellement de l'œuvre d'Eudes de Cheriton, fabuliste et prédicateur anglais originaire de Cheriton dans le Kent. Il était sans doute cistercien. Eudes vécut entre 1185 et 1246/1247. Il descendait d'une famille venue de Normandie après la victoire de Guillaume le Conquérant. Il visita Paris, où il étudia la théologie. Il séjourna en Espagne, dont il connut ainsi la culture. Le Libro de los gatos, collection de fables espagnoles du XIVe siècle, traduisent et amplifient bon nombre des fables d'Eudes de Cheriton, dont celle qui fait l'objet de la présente étude. L'œuvre d'Eudes de Cheriton occupe tout le tome 4 de l'ouvrage de Léopold Hervieux. Sur Eudes de Cheriton, sa vie et son activité, voir la synthèse d'Armijo Canto (3-16).

\section{LA FABLE ET LE PROVERBE}

Au cours des siècles, les auteurs ont tendu à développer le contenu moral et caché des fables qui, au départ étaient de simples récits. Parfois, la moralisation est introduite par l'adverbe mystice: 'je vais maintenant exposer le contenu caché (mysticum) de ma fable'. On entendait ainsi développer et expliciter la leçon de ces récits. Il semble que les fables primitives d'Esope ne comportaient pas d'introduction (promythion) ni de conclusion (épimythion) morales. Celles-ci étaient présentes, bien que brèves, dans la collection dite Augustana qui contient la première, mais tardive, anthologie des fables d'Ésope. Auparavant, Phèdre et Babrius, notamment, avaient développé cet enseignement moral, recourant largement aux proverbes moraux. Aux XIIIe et XIVe siècles, une volonté forte de moralisation des récits dans une perspective religieuse a remis la fable en honneur, l'accompagnant d'une critique acerbe des excès des puissants et des religieux. Cette critique est en particulier l'œuvre des ordres mendiants, si l'on en juge par l'origine de la plupart des auteurs des recueils d'exempla. ${ }^{1}$

Parmi les fables de Phèdre, Vulpis ad personam tragicam (éd. Brenot 6) est un texte très court, dépourvu de narration. En quatre vers, on ne trouve que l'évocation d'une situation, un propos de type parémique (dictum) et un épimythion de deux vers qui s'applique aussi bien à la fable qu'au proverbe:

1. Qu'on se reporte à la définition de l'exemplum médiéval par Le Goff, chez Bremond, Le Goff et Schmitt (30-38). 
Personam tragicam forte uulpes uiderat:

"o quanta species, inquit, cerebrum non habet!"

Hoc illis dictum est quibus honorem et gloriam

Fortuna tribuit, sensum communem abstulit.

"Un masque de tragédie vint à tomber sous les yeux du renard: $\ll \mathrm{O}$ la belle tête, s'écria le renard, pour n'avoir pas de cervelle!» Ce mot s'applique à certaines gens à qui le Fortune a accordé les honneurs et les sujets de gloriole, mais refusé le sens commun".

Le deuxième vers est une parémie, qui pourrait fort bien s'intégrer dans un court dialogue, une sorte de chreia dénonçant le peu de sagesse d'un individu riche et présomptueux. Ainsi procédaient Diogène et les philosophes cyniques.

\section{LA PENSÉE CYNIQUE}

La vraie richesse d'un individu est intérieure. La philosophie orientale, qu'on trouve contenue dans le Calila e Dimna, l'a montré: la sagesse est un trésor secret, réservé à quelques-uns. Ce recueil de fables réunies dans un cadre narratif est d'origine indienne (Panchatantra). Sa version en langue arabe, œuvre d'Ibn al-Muqaffa, constitue un maillon essentiel de la tradition. Sa version en langue espagnole (Calila e Dimna) est l'œuvre du roi Alphonse x le Sage. Elle est l'un des textes essentiels de la tradition exemplaire médiévale en Espagne. Dans le récit-cadre, le roi Sirechuel envoie le médecin Berzebuey en Inde en quête d'un savoir miraculeux. Au final, on découvre, comme le faisait Phèdre, que le trésor se trouve enfoui dans le cœur de l'homme avisé: "Homo doctus in se semper diuitias habet" (Phèdre, Fables IV 22) 'le vrai savant possède en lui-même sa richesse'.

Toute une littérature de type parémique s'est développée à partir des dialogues entre un philosophe et un homme plus puissant. Le philosophe peut être un cynique comme Diogène, un sage comme Aristote, un esclave comme Ésope, ou un enfant comme Secundo, Epictète, ou Teodor, la jeune fille. L'enfant est sage. Il enferme miraculeusement, malgré son jeune âge, toute la sagesse possible. Il s'agit là du topos du puer senex, dont Curtius, notamment, a étudié l'importance. ${ }^{2}$

2. Voir en particulier le chapitre 5 de Curtius: "La topique" (99-130). Ce topos confirme que la vérité ne vient pas de la plume des grands savants que la société honore: elle vient de la bouche des plus faibles: l'enfant, l'esclave, le modeste fabuliste ou le proverbe du vilain. 
L'influence de la philosophie cynique de Diogène, de Crates, de Bion ou de Ménippe sur les fables a été particulièrement soulignée par Rodríguez Adrados qui a réuni ses réflexions sur le sujet dans son ouvrage De Esopo al Lazarillo. ${ }^{3}$

Il n'est pas inutile d'observer que c'est grâce à Pierre Pithou, jusrisconsulte ami d'Henri de Navarre, qu'on doit la découverte du premier manuscrit des fables de Phèdre (1596). ${ }^{4}$ Or, Pierre Pithou est également le principal auteur de la Satire Ménippée, dirigée contre la Ligue et dont le nom est emprunté à Ménippe, philosophe cynique grec. La Réforme protestante devait aimer cette sagesse des cyniques, faite de modération et de refus de l'ostentatoire.

La sagesse du philosophe, quel qu'il soit, s'exprime simplement, sobrement, par la réponse à la devinette, par le proverbe, par la fable, par la chreia (qui est un court dialogue), par l'anecdote, par l'exemplum.

La fable et le proverbe se rejoignent en ce qu'ils expriment une sagesse pratique, concise, fondée sur des actes, plus que sur des paroles: le philosophe cynique se taisait et faisait montre de ses actes: on le connaît par sa vie (Ésope, Diogène, Secundo), ses exempla, ses dialogues, ses plaisanteries, ses paradoxes. Dans la Vie d'Esope, Ésope est l'esclave de maîtres successifs. Parmi eux figure Xanthos, un philosophe samien qui finit par reconnaître la sagesse supérieure de son esclave. ${ }^{5}$

La doxa reconnaît les bienfaits de l'argent, de la beauté, du pouvoir. $\mathrm{Pa}-$ radoxalement, l'Ésope de la Vita Aesopi est laid, pauvre et misérable. Il enseigne à se contenter de sa misère et à ne pas chercher à en sortir, à rester dans sa condition. Il tient des propos paradoxaux, hostiles au conformisme de la société. C'est ce que nous dit, en gros, le proverbe populaire. ${ }^{6}$ Comme le suggère le titre de l'ouvrage de Rodríguez Adrados, Lazarillo de Tormes, servi-

3. Rodríguez Adrados 295-376. Voir en particulier les chapitres suivants: "Elementos cínicos en las Vidas de Esopo y Secundo y en el Diálogo de Alejandro y los Gimnosofistas" (295-316), "Filosofía cínica en las fábulas esópicas" (317-28), "Política cínica en las fábulas esópicas" (329-40).

4. Voir l'édition citée de Robert Ulysse.

5. Voir l'article cité de Corinne Jouanno. Le texte est édité en espagnol par Bádenas de la Peña/ López Facal (163-282).

6. On se reportera à Silvia Palma. Lire en particulier le chapitre 5 "Les proverbes" (105-54). En particulier: "En tant que phrases génériques typifiantes a priori, les proverbes s'appuient sur une vérité de type général et nous postulons qu'ils peuvent soit focaliser sur la vérité générale, auquel cas ils font oublier l'existence éventuelle de contre-exemples, ce que nous appellerons le schéma doxal; soit souligner la présence de cas s'éloignant de la règle, ce que nous appellerons le schéma paradoxal" (134). Voir aussi une autre perspective dans Kleiber (142-56). 
teur de plusieurs maîtres successifs, apprend la sagesse par l'expérience de la servitude: il est le modèle du pícaro dont la sagesse est héritée d'une pratique de la vie. Ainsi est justifié le titre du livre de Rodríguez Adrados, De Esopo al Lazarillo.

Un très grand nombre d'œuvres médiévales est fondé sur l'enseignement d'un maître prodigué à un élève attentif, une forme de "miroir des princes", dont Le comte Lucanor, de don Juan Manuel est un bon exemple. Bien souvent, aussi, un philosophe, Aristote en particulier, conseille sagement un prince, l'empereur Alexandre dans le cas d'Aristote (Secreto de los secretos). Il s'agit là de formes notables du savoir: Patronio, le précepteur du comte Lucanor, est un sage reconnu. Aristote est un respectable philosophe. Il est d'ailleurs "le Philosophe", par antonomase. Face à ces savants, apparaît aussi sagesse une méprisée, celle de l'esclave qui révèle paradoxalement aux puissants la misère de leur condition et celle de l'enfant sage, qui étonne les plus grands et les plus doctes par l'étendue de son savoir infus.

Une œuvre archétypique (premier ou deuxième siècle après JésusChrist) de cette tendance est la Vie d'Esope, le fabuliste, dont l'existence est racontée dans un petit texte fait d'anecdotes et de paradoxes cyniques: un esprit porté sur la contradiction et une certaine grossièreté (Bádenas/López 166). Les deux versions du texte de la Vita Aesopi furent éditées en 1952 par B. E. Perry. Une des deux versions (le manuscrit G) fut traduite en espagnol en 2004 par Bádenas de la Peña et López Facal. Esope est un esclave qui va de maître en maître, jusqu'au philosophe Xanthos qui est finalement séduit par sa sagesse. L'esclave contrefait est laid extérieurement, mais sage intérieurement. Esope, prototype du pícaro, pratique une morale de vérité. Il traque et moque l'hypocrisie.

Bádenas (176) évoque des antécédents orientaux, comme la tradition d'Ahikar (Vie d'Abikar), conseiller de Sennaquerib, dont on connaît des versions syriaques, arabes et arméniennes, slaves et turques. Ahikar adopte $\mathrm{Na}$ dan, un de ses neveux, et l'enseigne par des sentences. Le neveu le calomnie et le roi condamne Ahikar à mort. Les rapports avec le Syntipas sont évidents. L'innocence d'Ahicar est plus tard reconnue, comme le sera celle de l'infant dans le Syntipas.

Il n'est pas inutile de relever que Phèdre est tenu, comme Esope, pour un esclave affranchi. Par ailleurs, le contenu philosophique et cynique des fables d'Esope ou de Phèdre se retrouve dans les proverbes, comme nous allons essayer de le décrire. 


\section{TeOdor ET EPicteto: LE topos DE L'Enfant SAGE}

Une bonne partie de cette sagesse du quotidien, faite de modestie et d'austérité se retrouve dans la tradition encyclopédique des livres d'énigmes et de devinettes, qu'Hugo O. Bizzarri a décrite dans son édition (1995) des dialogues entre Epictète et l'empereur Hadrien. On dit que le modèle revient aux foca Monachorum, traditions de devinettes et de sentences en honneur depuis le VIIIe siècle et largement inspirées par la tradition de l'Eglise, depuis la Bible, jusqu'aux Evangiles apocryphes (Acta Pilati, ou Evangile de Nicodème, en particulier). En espagnol, les textes les plus connus sont la Historia de la doncella Teodor, le Diálogo del filósofo Segundo y el emperador Adriano et le Diálogo de Epicteto y el emperador Adriano. Un texte fondateur est sans doute le premier Livre des Rois, où la Reine de Saba éprouve Salomon par des énigmes (I Rois 10: 1013): "Tu surpasses en sagesse et en prospérité la renommée dont j'ai eu l'écho".

La sagesse vient donc du philosophe (Socrate, Diogène, Esope...), mais aussi de l'Enfant Sage, selon un type folklorique très en vogue dans la culture occidentale, dont Curtius a fait un topos: le puer senex: l'enfant pourvu miraculeusement de la sagesse des anciens. Naturellement, Jésus, enseignant mieux que les scribes (Matthieu 7, 28; Marc 1, 21-22; et surtout Luc 2, 41-44), est l'exemple même de l'enfant sage.

On trouve ce topos de la philosophie des cyniques dans le livre de la Doncella Teodor, une encyclopédie à la fois scientifique, biblique et religieuse, mais tout autant dans la Vie d'Esope ou dans la parémiologie: l'idée que, moi homme, je ne suis rien, comme le penseront les pícaros. Peu importe donc le lieu d'où je viens. Aucun lieu ne surpasse l'autre et tous les hommes partagent la même condition.

On demande ainsi à l'enfant Péticus (Epicteto): "Omne, ¿de quál tierra sodes o dónde venides? Et él rrespondió: Yo so venido de mi padre et de mi madre, et so engendrado por voluntad de Dios" (Bizzarri 43). On demandait pareillement à Esope d'où il venait, lequel répondait: du ventre de ma mère. Bizzarri met le passage en relation avec le proverbe: "La tierra que me sé por madre me la he" (Santillana, Refranes, n. ${ }^{\circ}$ 396). On retrouve ce proverbe dans La lozana andaluza de Francisco Delicado, autre roman picaresque.

La Doncella Teodor? est un autre exemple de cet “enfant sage”, dont les réponses aux questions des sages constituent une forme d'encyclopédie. Son

7. Edition des deux textes dans Mettmann. A propos de Tawaddud, voir Gerresch. Le récit de "Tawaddud" occupe plus de 25 nuits, entre la 428ème et la 454ème. Autres études sur Tawaddud et Teodor: Bremond/Darbord; Darbord 1995. 
modèle arabe est la servante Tawaddud, qui dans les Mille et Une Nuits répond à des questions similaires. A côté d'un savoir théologique et scientifique, la cosmogonie et l'hygiène, Teodor et Tawaddud définissent un mode de vie simple et rigoureux, fermé aux excès de toutes sortes. Citons quelques-unes de ces questions (Mettmann 125-28):

19 El sabio le preguntó: "Donzella, qual es la cosa de la qual el hombre no se puede ver farto?". La doncella le respondió: "De ganar riquezas".

20 El sabio le preguntó: "Donzella, dime que cosa es hombre mancebo?". La doncella le respondió: "Candela encendida que luego se mata".

22 El sabio le preguntó: "Donzella, dime que cosa es más cierta?". La doncella le respondió: "La muerte de las personas".

24 El sabio le preguntó: "Donzella, por quantas cosas o maneras mienten los hombres?". La doncella le respondió: "Señor, el hombre miente por tres maneras: la primera o por deleyte de fablar, la segunda o por decir bien de quien bien quieren, la tercera o por decir mal de quien mal quieren".

C'est pour cela que les cyniques recommandaient le silence.

\section{LA SAGESSE DES FABLES}

Le tome 3 de la Historia de la fábula grecolatina de Francisco Rodríguez Adrados contient un inventaire du corpus des fables connues, séparé en trois types: $\mathrm{H}$ (Hausrath), no $\mathrm{H}$ et $\mathrm{M}$. Soit le corpus des fables anciennes $(\mathrm{H})$, les fables non intégrées dans ce corpus primitif (no $\mathrm{H}$ ) et enfin les fables médiévales, qu'on ne trouve pas avant le Romulus, les Ysopets, Marie de France, Vincent de Beauvais, Eudes de Cheriton, etc.

Nous sommes devant une tradition qui a réuni un ensemble fini de récits (1130), dont la caractéristique est qu'ils renvoient tous à une sagesse simple et pratique, semblable à celle des cyniques, mais aussi nourrie d'autres traditions, orientales en particulier. L'Inde connaissait les gymnosophistes dont la philosophie était peu différente de celle des cyniques. Autant de récits provenant peut-être d'un patrimoine commun très ancien, mésopotamien peut-être. Telle est l'hypothèse de Rodríguez Adrados. Si l'on relit les fables, on voit qu'elles enseignent une sagesse pratique que, pour notre part, nous résumons en cinq types: 
Type 1: Contre l'ambition des hommes. La richesse est trompeuse. Le vrai bonheur ne s'obtient pas avec elle. On voit comme ce thème a été exploité par les prédicateurs.

Type 2: Le puissant est un ingrat. Ses décisions sont arbitraires. Le pauvre est exploité.

Type 3: Vérité et mensonge. L'hypocrite se déguise pour tromper son prochain. Le mauvais conseil.

Type 4: L'homme est présomptueux. Il pêche par orgueil et jactance, par convoitise ou gourmandise.

Type 5, lié aux types précédents: L'habit ne fait pas le moine, "Genio y figura, hasta la sepultura". L'homme a beau se déguiser, il sera toujours le même. Il faut accepter sa condition.

\section{DE LUPO QUI VOLUIT ESSE MONACHUS}

A titre d'illustration de ce qui précède, et pour montrer que la sagesse est exprimée par la sentence et par la fable, nous avons choisi de présenter ici pour terminer la fable médiévale du loup devenu moine, d'Eudes de Cheriton. Pour la raconter, le clerc recourt à des proverbes, ce qui confirme notre hypothèse: fable et parémie sont en relation paraphrastique. Elles enseignent une même sagesse.

Voici la version d'Eudes de Cheriton: ${ }^{8}$

OC XXII De Lupo qui uoluit esse monachus. Contra malam consuetudinem.

Ysemgrinus semel uoluit esse monachus. Magnis precibus optinuit quod Capitulum consensit; coronam, cucullam et cetera monachalia suscepit. Tandem posuerunt eum ad litteras; debuit addiscere Pater Noster, et semper respondit Agnus uel Aries. Docuerunt eum ut respiceret ad Crucifixum, ad sacrificium, et ille semper direxit oculos ad arietes.

Sic plerique fiunt monachi. Semper tamen dicunt Aries, semper clamant bonum uinum, semper habent oculum ad pingue frustum, ad scutellam suam. Vnde solet dici: Thai thu Wolf hore bodi te preste yho thu bym sette Salmes to lere, ever beth his geres to the groue-ward. Similiter, si senem fatuum et insensatum uelis instruere, nunquam relinquis antiquum morem. Ve-

8. OC pour Eudes (Odo) de Cheriton, LG pour Libro de los gatos (editado en Darbord 1984). 
tus retorta frangi potest, plicari non potest: uetus runcinus nunquam addiscit ambulare. Item quidam sunt ita asinine nature, quod nunquam uolunt antiquam consuetudinem dimittere. Unde: Pectina asinum, ablue asinum, rade asinum, nunquam perduces asinum ad bonum equum. Jeremias (13, 23): Si potest pardus mutare uarietatem suam, et ethiops pellem suam, et uos poteritis bona agere, cum didiceritis male; quoniam equus retinet in natura quod didicit in domitura. Difficile est consueta relinquere: Sordibus imbuti nequeunt dimittere sordes.

Les formes parémiques apparaissent en gras. Voici maintenant la traduction espagnole de cette fable, contenue dans le Libro de los gatos. (Darbord 1984, 80-81)

LG XIX Enxiemplo del lobo con los monges

El lobo una vegada quiso ser monge, e rogó a un convento de monges que lo quisiesen y rescebir; e los monges ficiéronlo ansí, e ficieron al lobo la corona, e diéronle cugulla e todas las otras cosas que pertenescen al monge, e pusiéronle a ller Pater Noster. Él, en lugar de decir Pater Noster, siempre decía cordero o carnero, e decíanle que parase mientes al crucifixo e al cuerpo de Dios. Él siempre catava al cordero o al carnero. Bien ansí acaesce a muchos monges que, en lugar de aprender la regla de la orden de las cosas que pertenescen a Dios, siempre responden e llaman carnero, que se entiende por las buenas viandas e por el vino, e por otros vicios d'este mundo.

Esto mismo se entiende en este enxiemplo por algunos viejos que son envegecidos en mal, e en locura e en malas constumbres. Onde por mucho que otro los castigue, nunca quieren dexar sus viejas costumbres. Onde el ombre viejo antes lo podrás quebrantar que non doblar. Faz all asno buena silla e buen freno quanto bien podieres, e nunca podras d'él facer buen cavallo en quanto vivas.

Le loup s'est déguisé en moine, mais il ne peut oublier sa vraie condition, ses vrais penchants. On retrouve là notre type $n .^{\circ} 5$. Un dicton espagnol dit que la chèvre est toujours tournée vers les endroits sauvages où elle a grandi: $L a$ cabra siempre tira al monte. La parémiologie a largement exploité le domaine et le texte d'Eudes le montre bien. La fable d'Esope correspondante traitait de ce simple thème cynique: il faut se contenter de ce que l'on est, de ce que l'on a, sans autre ambition. Chez Eudes ou chez l'auteur espagnol, le loup figure les moines et les clercs hypocrites, qui n'ont choisi la vie religieuse que pour vivre confortablement. Notre ambition (type 1) est au fond le déni de notre 
vraie nature (type 5), une forme de mensonge ou de déguisement (type 3), d'ingratitude (type 2) et de présomption (type 4).

Chez Eudes, la fonction paraphrastique du proverbe a souvent une dimension diaphasique. Le clerc s'exprime gravement, mais il recourt parfois au proverbe pour adopter un registre plus léger, plus populaire. Il abandonne même le latin parfois et exploite la langue vulgaire, le vieil anglais. Il interroge aussi la Bible en latin, et multiplie les adages, plus que son traducteur espagnol, au siècle suivant. Eudes aime donc intercaler des proverbes en langue vulgaire, en français ou, comme ici, en anglais. L'anglais était la langue du peuple, peu écrite, à la différence du français, sans doute mieux considérée puisque importée par des conquérants de plus haute culture.

Les autres parémies permettent de rapprocher fables et proverbes. Tous ces énoncés exploitent un bestiaire très stéréotypé. Comme dans la fable, on sépare bestia (la bête sauvage) et pecus (l'animal domestique), et parmi le pecus les jumenta et armenta (Dittmar 421-32). L'animal sauvage (bestia) ne peut être dompté et éduqué: le loup, dans la fable et dans le proverbe en vieil anglais. Le léopard (pardus) et la panthère noire (ethiops) dans le proverbe qui suit ne changeront jamais: on peut changer la couleur de leur peau, ils resteront ce qu'ils sont, des bêtes sauvages.

Nous sommes là devant un motif extrêmement productif: un grand nombre des fables d'Eudes en est issu:

OC XV De cato qui se fecit Monachum: le chat se fait moine pour manger les souris du monastère. Il se fait tonsurer et enfile un habit.

OC XXIIIa Quidam commendavit XII oves compatri suo lupo. Un loup dévore les douze brebis qu'on lui a confiées. Il est dénoncé par ses dents ensanglantées.

OC XXVI De asinis indutis pellibus leoninis. Les ânes revêtent une peau de lion pour effrayer les hommes. Mais ils sont dénoncés par leur voix d'ânes. OC L De vulpe et gallinis. Le renard veut pénétrer dans le poulailler. Il fait croire aux poules qu'il est affaibli et inoffensif. Les poules le laissent entrer.

OC LI De fraude vulpis. Un renard revêt une peau de brebis pour s'approcher du troupeau.

9. Pecus, n. au pluriel pecua, désigne le troupeau et, par extension l'argent que celui-ci représente (pecunia). Pecus, pecoris, n., désigne le troupeau, pecus, pecudis, f., désigne la tête individuelle de bétail et devient facilement un terme d'injure. Voir le dictionnaire d'Ernout et Meillet. 
OC LIa De fraude comitis. Un comte félon rançonne les voyageurs. Pour ne pas effrayer ceux qui s'approchent, il revêt l'habit des moines de Citeaux. OC LII De contentione ovis albe et ovis nigre, asini et hirci. La brebis blanche, la brebis noire, l'âne et le bouc discutent de leur habit. Chacun figure allégoriquement les moines blancs, noirs, ceux qui portent la croix ou une longue barbe. Tout, pourtant n'est qu'apparence.

OC XLIX De vulpe. Pour dévorer les oiseaux, le renard fait le mort et tire la langue. Les oiseaux s'approchent alors sans méfiance.

Le loup et le renard sont des bestiae, des bêtes sauvages. On voit que dans le rang des pecuaria, les ânes et les chevaux (asini, runcini, equi) sont également propices à cette leçon: nunquam relinquis antiquum morem. Vetus retorta frangi potest, plicari non potest. 'Jamais tu n'abandonneras ta vraie nature, et tu casseras, avant que quelqu'un ne t'ait fait plier'. "Vetus runcinus nunquam addiscit ambulare". 'Tu ne mettras jamais au pas un vieux cheval, s'il ne l'a jamais fait de sa vie'. Item quidam sunt ita asinine nature, quod nunquam uolunt antiquam consuetudinem dimittere. Unde: Pectina asinum, ablue asinum, rade asinum, nunquam perduces asinum ad bonum equum. 'Tu peux toujours brosser, laver, raser ton âne, jamais tu n'en feras un bon cheval'.

Ce dernier énoncé (Pectina asinum...) est un proverbe, fait de trois impératifs successifs, réunis par un même accusatif (asinum) trois fois répété, suivi d'une apodose opposant le mauvais et le bon serviteur (asinum vs equum). Cet énoncé est un proverbe par son connecteur unde et par la ponctuation qui le démarque.

Cette commune participation de l'animal dans le proverbier et dans la fable incline à étudier le bestiaire médiéval et la fonction de chaque animal. En ce qui concerne la langue espagnole, l'étude du refranero par Alexandra Oddo, ou celle, plus ancienne, d'Elizabeth O'Kane, peuvent nous y aider. Oddo a confronté en diachronie les proverbiers médiévaux espagnol. O'Kane, pour sa part, a répertorié les phrases proverbiales de la littérature médiévale espagnole en en indexant le vocabulaire (gato, león, lobo...).

\section{TEL EN PENSÉE, TEL EN LA BOUCHE}

Un proverbe n'est rien sans une situation qui le détermine et l'éclaire. Tous les conteurs et les fabulistes utilisent le proverbe, ou le dicton, ou la phrase situationnelle, pour justifier ou illustrer leur récit. Le proverbe a une fonction mémorielle. Telle une illustration (bistoria), il sert à marquer les esprits. C'est 
pourquoi un proverbe conclut souvent une fable. Ainsi procédait Marie de France qui, comme Eudes de Cheriton, a largement puisé dans la tradition ésopique. Pour conclure, j'aimerais rappeler la fable de Marie de France pareillement consacrée à ce motif du loup qui n'arrive pas à déguiser complètement sa mauvaise intention. On verra comme Marie de France conclut sa fable par ce qui est, par sa structure syntaxique et rythmique, un proverbe: Tel en pensé, tel en la buche (Boivin/Harf-Lancner 118-19):

\section{Le prêtre et le loup}

Uns prestre volt jadis apprendre un lou a letres faire entendre. "A" dist li prestre, "a" dist li lous, ki mult ert fel e engignous. "B" dist li prestre, "di od mei!" "B" dist li lous, "la letre vei". "C" dist li prestre, "di avant!" "C" dist li lous, "a I dunc tant?" Respunt li prestre: "Or di par tei!" Li loup li dist: "Jeo ne sai quei". "Di que te semble, si espel!" Respunt li lous: "Aignel, aignel!" Li prestre dist que verté tuche: tel en pensé, tel en la buche. De plusurs le veit hum sovent: cel dunt il pensent durement est par lur buche cuneü, anceis que d'altre seit seü; la buche mustre le penser, tut deië ele d'el parler.
Un prêtre voulut un jour apprendre a un loup à lire l'alphabet.

"A" dit le prêtre. "A", dit le loup, qui était cruel et rusé. "B", dit le prêtre, "répète avec moi!" -"B", dit le loup, "je vois la lettre". -"C", dit le prêtre, "répète-le!" _"C", dit le loup, "il y en a donc tant?" Le prêtre répond: "A toi maintenant!" Le loup lui dit: "Je ne sais quoi dire". -"Dis ce qui te vient à l'esprit, épelle-le!" Le loup répond: "Agneau, agneau!" Le prêtre dit qu'il touche au vrai: sa parole répond à sa pensée.

On le voit pour bien des gens:

l'objet de leurs pensées est découvert par leur bouche avant tout autre; la bouche révèle la pensée, même si on veut lui faire dire autre chose.

\section{CONCLUSION: À PROPOS DES PARADOXES PROVERBIAUX}

Après ce passage des fables et proverbes au crible de la pensée des cyniques, on peut en conclusion s'interroger sur la place de cette forme de pensée par rapport à la doxa et à l'équilibre d'une société. Certaines fables prônent l'acceptation de l'ordre établi: tu as ta nature, tu occupes un état. Reste-z-y! Le loup ne doit pas se déguiser en moine, car il n'en a pas les moyens: "tel en pen- 
sée, tel en la bouche" suivrait à ce sujet le schéma doxal qu'a décrit Silvia Palma. En revanche, la fable et le proverbe, en ce qu'ils défendent une pauvreté et une laideur apparente, en ce qu'ils expriment des sentences opposées aux lieux communs de la société, en ce qu'ils se donnent pour sages, des esclaves ou des mendiants difformes comme Diogène ou Ésope, peuvent exprimer un savoir ou une éthique subversifs. Le bien est chose inattendue, loin de ce qu'on pense.

\section{OUVRAGES CITÉS}

Armijo Canto, Carmen Elena. Fábula y mundo: Odo de Chériton y el Libro de los gatos. México: UnAM, 2014.

Bádenas de la Peña, Pedro y Javier López Facal, trad. y notas. Fábulas de Esopo. Vida de Esopo. Fábulas de Babrio. Intr. Carlos García Gual. 3a ed. Madrid: Gredos, 2004.

Bizzarri, Hugo O., ed. Diálogo de Epicteto y el emperador Adriano. Madrid: Iberoamericana, 1995.

Bizzarri, Hugo O. "La passage du proverbe à l'exemplum et de l'exemplum au proverbe". Tradition des proverbes et des exempla dans l'Occident médiéval. Berlin: De Gruyter, 2009. 9-23.

Boivin, Jeanne-Marie et Laurence Harf-Lancner, éds. Fables françaises du Moyen Age: les Isopets (édition bilingue). Paris: Garnier-Flammarion, 1996.

Bremond, Claude et Bernard Darbord. "Tawaddud et Teodor: les enjeux ludiques du savoir”. L'enciclopedismo Medievale. Ed. Michelangelo Picone. Ravenna: Longo, 1994. 253-73.

Bremond, Claude, Jacques Le Goff et Jean-Claude Schmitt. L'Exemplum. Turnhout: Brepols, 1982.

Calila e Dimna. Eds. Juan Manuel Cacho Blecua y María Jesús Lacarra. Madrid: Castalia, 1984.

Carron, Delphine. "Présence de Caton dans les proverbes et les exemples médiévaux". Tradition des proverbes et des exempla dans l'Occident médiéval. Éds. Hugo O. Bizzarri et Martin Rhode. Scrinium Friburgense. Berlin: Walter de Gruyter, 2009. 165-90.

Curtius, Ernst Robert. La Littérature européenne et le Moyen Age Latin. Paris: Presses Universitaires de France, 1956.

Darbord, Bernard. Libro de los gatos. Annexes des Cahiers de Linguistique Hispanique Médiévale 3. Paris: Klincksieck, 1984. 
Darbord, Bernard. "La tradición del saber en la Doncella Teodor". Medioevo y Literatura (Actas del V Congreso de la Asociación Hispánica de Literatura Medieval). Vol. 1. Ed. Juan Paredes. Granada: Universidad de Granada, 1995. 13-30.

Dittmar, Pierre-Olivier. "L'animal, l'humain et les images". Les Images dans l'Occident médiéval. Éds. Jérôme Baschet et Pierre-Olivier Dittmar. Turnhout: Brepols, 2015. 421-32.

Ernout, Alfred et Antoine Meillet. Dictionnaire étymologique de la langue latine: Histoire des mots. 4ème éd. Paris: Klincksieck, 1985.

Gerresch, Claudine. "Un récit des Mille et Une Nuits: Tawaddud. Petite encyclopédie de l'Islam médiéval". Bulletin de l'Institut Français d'Afrique Noire 25, série B, n. ${ }^{\circ} 1$ (1973): 57-175.

Herrmann, Léon. Phèdre et ses fables. Leiden: Brill, 1950.

Hervieux, Leopold. Les Fabulistes Latins depuis le siècle d'Auguste jusqu'à la fin du moyen âge. Vol. 5. Paris: Firmin Didot, 1899. Reimp. Hildesheim: Georg Olms, 1970.

Jouanno, Corinne. "La Vie d'Esope: une biographie comique". Revue des Études Grecques 118.2 (2005): 391-425. http://www.persee.fr/web/revues/home/ prescript/article/reg_0035-2039_2005_num_118_2_4633.

Juan Manuel. El conde Lucanor. Ed. Guillermo Serés. Barcelona: Crítica, 1994. Kleiber, Georges. "Sur le chemin des proverbes, questions de classification". Voix et marqueurs de discours: des connecteurs à l'argument d'autorité. Dirs. Jean-Claude Anscombre, Amalia Rodríguez Somolinos et Sonia GómezJordana Ferary. Lyon: ENS Editions, 2012. 141-63.

López de Mendoza, Íñigo, Marqués de Santillana. Refranes que dizen las viejas tras el fuego. Ed. Hugo O. Bizzarri. Kassel: Reichenberger, 1995.

Mettmann, Walter, ed. La historia de la donzella Teodor. Ein spanisches Volksbuch arabischen Ursprungs. Mainz: Akademie der Wissenschaften und der Lieratur, 1962.

Oddo, Alexandra. Vers un refranero diacbronique: Analyse linguistique de l'évolution des proverbes espagnols depuis le Moyen Age. Limoges: Lambert-Lucas, 2013.

O'Kane, Eleanor. Refranes y frases proverbiales españolas de la Edad Media. Anejo 2 del Boletín de la Real Academia Española. Madrid: RAE, 1959.

Palma, Silvia. Les Éléments figés de la langue: Étude comparative français-espagnol. Paris: L'Harmattan, 2007.

Perry, Ben E. Aesopica, Urbana: Illinois UP, 1952.

Phèdre. Fables. Ed. Alice Brenot. Paris: Les Belles Lettres, 1961. 
Rodríguez Adrados, Francisco. De Esopo al Lazarillo. Huelva: Universidad de Huelva, 2005.

Secreto de los secretos. Ed. Hugo Oscar Bizzarri. 23 de noviembre de 2017. $<$ http://filosofia.org/aut/001/secreto.htm>.

Ulysse, Robert. Les Fables de Phèdre: Édition paléographique publiée d'après le manuscrit Rosanbo. Paris: Imprimerie nationale, 1893. 